\title{
ON THE CONVERGENCE OF DOMAIN DECOMPOSITION ALGORITHM FOR THE BODY WITH THIN INCLUSION
}

\author{
Andriy STYAHAR*, Yarema SAVULA* \\ ${ }^{*}$ Faculty of Applied Mathematics and Informatics, Department of Applied Mathematics, \\ Ivan Franko Lviv National University, Universytetska,1, 79000, Lviv, Ukraine \\ astyahar@gmail.com, savula@franko.lviv.ua
}

\begin{abstract}
We consider a coupled 3D model that involves computation of the stress-strain state for the body with thin inclusion. For the description of the stress-strain state of the main part, the linear elasticity theory is used. The inclusion is modelled using Timoshenko theory for shells. Therefore, the dimension of the problem inside the inclusion is decreased by one. For the numerical solution of this problem we propose an iterative domain decomposition algorithm (Dirichlet-Neumann scheme). This approach allows us to decouple problems in both parts and preserve the structure of the corresponding matrices. We investigate the convergence of the aforementioned algorithm and prove that the problem is well-posed.
\end{abstract}

Key words: Elasticity Theory, Timoshenko Shell Theory, Steklov-Poincare Operator, Domain Decomposition

\section{INTRODUCTION}

A lot of structures, that occur in engineering, are inhomogeneous and contain thin parts and massive parts. Therefore, it is important to develop both analytical methods and numerical algorithms for the analysis of the stress-strain state of such structures.

Different aspects of such problems were discussed in Dyyak et al. (2012); Niemi et al. (2010); Savula et al. (2000); Vynnytska and Savula (2008); Nazarov (2005) (in Vynnytska and Savula (2008) the case of the bodies with thin inclusions is considered; in Dyyak et al. (2012) the bodies with thin covers are considered; in Nazarov (2005) asymptotic methods are used for the analysis of the elastic bodies with thin rods). Papers Niemi et al. (2010) and Savula et al. (2000) are devoted to the numerical solution of the Girkmann problem. The discussion on the problems of thermoelasticity the reader may find in Sulym (2007).

In this article, we consider a model for the description of the stress-strain state for the 3D body with thin inclusion. The main part of the body is modelled using the linear elasticity theory. The thin part is modelled using the Timoshenko theory for shells. In order to numerically solve this problem, we propose an iterative domain decomposition algorithm which connects solutions in both parts using coupling conditions. We prove the convergence of the proposed algorithm and the existence and uniqueness of the solution of the corresponding Steklov-Poincare interface equation.

The application of domain decomposition method allows us to decouple problems in both parts and solve the problems independently in each part. As a result, it is possible to compute the stress-strain state accurately even for small shell thicknesses without having problems with stability issues of the coupled problem.

\section{PROBLEM STATEMENT}

Let us consider a problem of a stress-strain state of an elastic body $\Omega_{1}$ with the inclusion in $\Omega_{2}$ (Fig. 1).

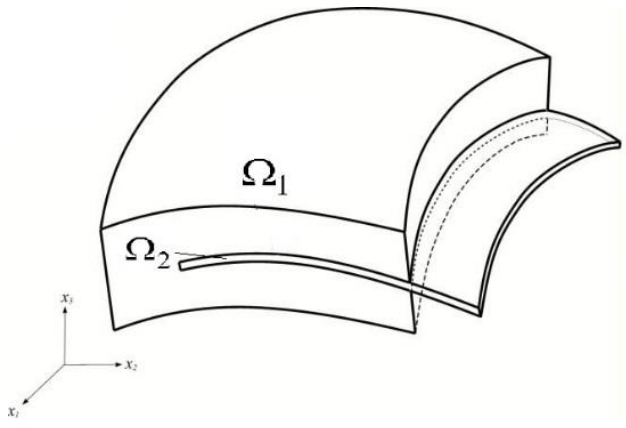

Fig. 1. Body with inclusion

Let us describe the stress-strain state of the body in $\Omega_{1}$ in rectangular coordinate system $x_{1}, x_{2}, x_{3}$ using the theory of linear elasticity. Let us denote by $\Sigma=\left(\sigma_{i j}\right)_{i, j=1}^{3}$ the Cauchy stress tensor. The components of $\Sigma$ are found from the relationships

$\sigma_{i j}=\frac{1}{2} E_{1}\left(\frac{\partial u_{i}}{\partial x_{j}}+\frac{\partial u_{j}}{\partial x_{i}}\right), i, j=1,2,3$,

where: $E_{1}$ is the Young's modulus of the body in $\Omega_{1} ; \mathrm{u}(\mathrm{x})=$ $\left(u_{1}(x), u_{2}(x), u_{3}(x)\right)$ is the displacement vector with $u_{i}$ being the displacements along the directions of $x_{i}, i=1,2,3$.

Equilibrium equations for the body in $\Omega_{1}$ have the form:

$\frac{\partial \sigma_{11}}{\partial x_{1}}+\frac{\partial \sigma_{12}}{\partial x_{2}}+\frac{\partial \sigma_{13}}{\partial x_{3}}=f_{1}$,

$\frac{\partial \sigma_{21}}{\partial x_{1}}+\frac{\partial \sigma_{22}}{\partial x_{2}}+\frac{\partial \sigma_{23}}{\partial x_{3}}=f_{2}$,

$\frac{\partial \sigma_{31}}{\partial x_{1}}+\frac{\partial \sigma_{32}}{\partial x_{2}}+\frac{\partial \sigma_{33}}{\partial x_{3}}=f_{3}$,

where: $\mathrm{x} \in \Omega_{1}, \mathrm{f}=\left(f_{1}, f_{2}, f_{3}\right)$ is the vector of volume forces.

In the following we assume that no volume forces act on the body in $\Omega_{1}$.

Let us denote by $\mathrm{n}=\left(n^{1}, n^{2}, n^{3}\right)$ outer normal vector and by $\tau_{1}=\left(\tau_{1}^{1}, \tau_{1}^{2}, \tau_{1}^{3}\right), \tau_{2}=\left(\tau_{2}^{1}, \tau_{2}^{2}, \tau_{2}^{3}\right)$ corresponding tangent vectors. 
Equations (1) are supplemented by the boundary conditions of one of the following types.

Kinematic (Dirichlet) boundary conditions are of the form:

$$
u_{n}=u_{n}^{0}, u_{\tau_{1}}=u_{\tau_{1}}^{0}, u_{\tau_{2}}=u_{\tau_{2}}^{0}, \mathrm{x} \in \Gamma_{1} \text {, }
$$

where: $\Gamma_{1}$ is the outer boundary of $\Omega_{1} ; u_{n}, u_{\tau_{1}}$ and $u_{\tau_{2}}$ are the components of the displacement vector in the coordinate system $\mathrm{n}, \tau_{1}, \tau_{2} ; u_{n}^{0}, u_{\tau_{1}}^{0}$ and $u_{\tau_{2}}^{0}$ are the prescribed displacements on $\Gamma_{1}$.

Static (Neumann) boundary conditions have the form:

$\sigma_{n n}=\sigma_{n n}^{0}, \sigma_{n \tau_{1}}=\sigma_{n \tau_{1}}^{0}, \sigma_{n \tau_{2}}=\sigma_{n \tau_{2}}^{0}, \mathrm{x} \in \Gamma_{1}$,

where: $\sigma_{n n}, \sigma_{n \tau_{1}}$ and $\sigma_{n \tau_{2}}$ are the components of the stress tensor in the coordinate system; $\mathrm{n}, \tau_{1}, \tau_{2} ; \sigma_{n n}^{0}, \sigma_{n \tau_{1}}^{0}$ and $\sigma_{n \tau_{2}}^{0}$ are the prescribed stresses on $\Gamma_{1}$.

It is possible to consider other types of boundary conditions, for example mixed boundary conditions, that combine boundary conditions (2) and (3).

For the description of the stress-strain state of the inclusion $\Omega_{2}$ we use the equations of Timoshenko shell theory in the curvilinear coordinate system $\left(\xi_{1}, \xi_{2}, \xi_{3}\right)$ that hold on the median surface $\Omega_{2}^{*}$, where: $\Omega_{2}=\left\{\left(\xi_{1}, \xi_{2}, \xi_{3}\right): \xi_{1}^{b} \leq \xi_{1} \leq \xi_{1}^{e}, \xi_{2}^{b} \leq\right.$ $\left.\xi_{2} \leq \xi_{2}^{e},-\frac{h}{2} \leq \xi_{3} \leq \frac{h}{2}\right\}, \quad h$ is the thickness of the inclusion in $\Omega_{2}$.

By $\Omega_{2}^{*}$ we denote the median surface of $\Omega_{2}$ (the projection of $\Omega_{2}$ on the surface for which $\xi_{3}=0$ ).

The equations of Timoshenko shell theory are of the form (Pelekh, 1978):

$$
\begin{aligned}
& \frac{1}{A_{1} A_{2}} \frac{\partial\left(A_{2} T_{11}\right)}{\partial \xi_{1}}-\frac{1}{A_{1} A_{2}} \frac{\partial A_{2}}{\partial \xi_{1}} T_{22}+\frac{1}{A_{1}^{2} A_{2}} \frac{\partial\left(A_{1}^{2} T_{12}\right)}{\partial \xi_{2}}+ \\
& +k_{1} T_{13}+\frac{1}{A_{1} A_{2}} \frac{\partial\left(\frac{A_{1}}{r_{1}} M_{11}\right)}{\partial \xi_{2}}+\frac{k_{2}}{A_{1} A_{2}} \frac{\partial A_{1}}{\partial \xi_{2}} M_{12}= \\
& =-\left(p_{1}^{+}+p_{1}^{-}\right), \\
& -\frac{1}{A_{1} A_{2}} \frac{\partial A_{1}}{\partial \xi_{2}} T_{11}+\frac{1}{A_{1} A_{2}} \frac{\partial\left(A_{1} T_{22}\right)}{\partial \xi_{2}}+\frac{1}{A_{1} A_{2}^{2}} \frac{\partial\left(A_{2}^{2} T_{12}\right)}{\partial \xi_{1}}+ \\
& +k_{2} T_{23}+\frac{1}{A_{1} A_{2}} \frac{\partial\left(\frac{A_{2}}{r_{2}} M_{22}\right)}{\partial \xi_{2}}+\frac{k_{1}}{A_{1} A_{2}} \frac{\partial A_{2}}{\partial \xi_{1}} M_{12}= \\
& -k_{1} T_{11}-k_{2} T_{22}+\frac{1}{A_{1} A_{2}} \frac{\partial\left(A_{2} T_{13}\right)}{\partial \xi_{1}}+\frac{1}{A_{1} A_{2}} \frac{\partial\left(A_{1} T_{23}\right)}{\partial \xi_{2}}= \\
& -T_{13}+\frac{1}{A_{1} A_{2}} \frac{\partial\left(A_{2} M_{11}\right)}{\partial \xi_{1}}-\frac{1}{A_{1} A_{2}} \frac{\partial A_{2}}{\partial \xi_{1}} M_{22}+ \\
& +\frac{1}{A_{1}^{2} A_{2}} \frac{\partial\left(A_{1}^{2} M_{12}\right)}{\partial \xi_{2}}=-\frac{h}{2}\left(p_{1}^{+}-p_{1}^{-}\right), \\
& -T_{23}-\frac{1}{A_{1} A_{2}} \frac{\partial A_{1}}{\partial \xi_{2}} M_{11}+\frac{1}{A_{1} A_{2}} \frac{\partial\left(A_{1} M_{22}\right)}{\partial \xi_{2}}+ \\
& +\frac{1}{A_{1} A_{2}^{2}} \frac{\partial\left(A_{2}^{2} M_{12}\right)}{\partial \xi_{1}}=-\frac{h}{2}\left(p_{2}^{+}-p_{2}^{-}\right),
\end{aligned}
$$

where: $T_{11}, T_{12}, T_{22}, T_{13}, T_{23}, M_{11}, M_{12}, M_{22}$ are the forces and momenta in the shell; $A_{1}=A_{1}\left(\xi_{1}, \xi_{2}\right), A_{2}=A_{2}\left(\xi_{1}, \xi_{2}\right), k_{1}=$ $k_{1}\left(\xi_{1}, \xi_{2}\right), k_{2}=k_{2}\left(\xi_{1}, \xi_{2}\right)$ correspond to Lame parameters and median surface curvature parameters; $r_{1}=\frac{1}{k_{1}}, r_{2}=\frac{1}{k_{2}}$; $\left(\xi_{1}, \xi_{2}\right) \in \Omega_{2}^{*} ; p_{1}^{+}, p_{1}^{-}, p_{2}^{+}, p_{2}^{-}, p_{3}^{+}, p_{3}^{-}$are given functions; it holds:

$$
\begin{aligned}
& T_{\alpha \alpha}=\frac{E_{2} h}{\left(1-\vartheta_{2}^{2}\right)}\left(\varepsilon_{\alpha \alpha}+\vartheta_{2} \varepsilon_{\beta \beta}\right) ; T_{12}=\frac{E_{2} h}{2\left(1+\vartheta_{2}\right)} \varepsilon_{12} \\
& T_{\alpha 3}=k^{\prime} G^{\prime} h \varepsilon_{\alpha 3} ; \\
& M_{\alpha \alpha}=\frac{E_{2} h^{3}}{12\left(1-\vartheta_{2}^{2}\right)}\left(\chi_{\alpha \alpha}+\vartheta_{2} \chi_{\beta \beta}\right) ; M_{12}=\frac{E_{2} h^{3}}{12\left(1+\vartheta_{2}\right)} \chi_{12}
\end{aligned}
$$

where: $\alpha, \beta=1,2, \alpha \neq \beta ; k^{\prime}, G^{\prime}$ are constants that characterize transversely isotropic material; $E_{2}$ is the Young's modulus of the shell, $\vartheta_{2}$ is the Poisson's ratio.

The strains $\varepsilon_{11}, \varepsilon_{22}, \varepsilon_{12}, \varepsilon_{13}, \varepsilon_{23}, \chi_{11}, \chi_{22}, \chi_{12}$ are obtained from the relationships:

$$
\begin{aligned}
& \varepsilon_{\alpha \alpha}=\frac{1}{A_{\alpha}} \frac{\partial \mathrm{v}_{\alpha}}{\partial \xi_{\alpha}}+\frac{1}{A_{\alpha} A_{\beta}} \mathrm{v}_{\beta} \frac{\partial \mathrm{A}_{\alpha}}{\partial \xi_{\beta}}+k_{\alpha} w, \\
& 2 \varepsilon_{\alpha \beta}=\frac{A_{\alpha}}{A_{\beta}} \frac{\partial \frac{\mathrm{v}_{\alpha}}{A_{\alpha}}}{\partial \xi_{\beta}}+\frac{A_{\beta}}{A_{\alpha}} \frac{\partial \frac{\mathrm{v}_{\beta}}{A_{\beta}}}{\partial \xi_{\alpha}} ; \varepsilon_{\alpha 3}=-k_{\alpha} \mathrm{v}_{\alpha}+\frac{1}{A_{\alpha}} \frac{\partial w}{\partial \xi_{\alpha}}+\gamma_{\alpha} \\
& \chi_{\alpha \alpha}=\frac{1}{A_{\alpha}} \frac{\partial \gamma_{\alpha}}{\partial \xi_{\alpha}}+\frac{1}{A_{\alpha} A_{\beta}} \gamma_{\beta} \frac{\partial \mathrm{A}_{\alpha}}{\partial \xi_{\beta}} \\
& 2 \chi_{\alpha \beta}=\frac{k_{\alpha}}{A_{\beta}} \frac{\partial \mathrm{v}_{\alpha}}{\partial \xi_{\beta}}-\frac{k_{\beta}}{A_{\alpha} A_{\beta}} \mathrm{v}_{\alpha} \frac{\partial A_{\alpha}}{\partial \xi_{\beta}}+\frac{k_{\beta}}{A_{\alpha}} \frac{\partial \mathrm{v}_{\beta}}{\partial \xi_{\alpha}}- \\
& -\frac{k_{\alpha}}{A_{\alpha} A_{\beta}} \mathrm{v}_{\beta} \frac{\partial A_{\beta}}{\partial \xi_{\alpha}}+\frac{A_{\alpha}}{A_{\beta}} \frac{\partial \frac{\gamma_{\alpha}}{A_{\alpha}}}{\partial \xi_{\beta}}+\frac{A_{\beta}}{A_{\alpha}} \frac{\partial \frac{\gamma_{\beta}}{A_{\beta}}}{\partial \xi_{\alpha}}
\end{aligned}
$$

where: $\alpha, \beta=1,2, \alpha \neq \beta ; \mathrm{v}_{1}=\mathrm{v}_{1}\left(\xi_{1}, \xi_{2}\right), \mathrm{v}_{2}=\mathrm{v}_{2}\left(\xi_{1}, \xi_{2}\right)$, $w=w\left(\xi_{1}, \xi_{2}\right), \quad \gamma_{1}=\gamma_{1}\left(\xi_{1}, \xi_{2}\right), \quad \gamma_{2}=\gamma_{2}\left(\xi_{1}, \xi_{2}\right)$ are the displacements and angles of revolution in the shell;

$$
\begin{aligned}
& p_{i}^{+}=\left(1+k_{1} \frac{h}{2}\right)\left(1+k_{2} \frac{h}{2}\right) \sigma_{i 3}^{+}, \\
& p_{i}^{-}=\left(1-k_{1} \frac{h}{2}\right)\left(1-k_{2} \frac{h}{2}\right) \sigma_{i 3}^{-}, i=1,2,3 .
\end{aligned}
$$

Here $\sigma_{i 3}^{+}, \sigma_{i 3}^{-}, i=1,2,3$ are the components of the stress tensor on the top $\left(\xi_{3}=\frac{h}{2}\right)$ and bottom $\left(\xi_{3}=-\frac{h}{2}\right)$ surfaces of the shell. It is known that in the case of isotropic bodies we have $k^{\prime}=\frac{5}{6}, G^{\prime}=\frac{E_{2}}{2\left(1+\vartheta_{2}\right)}$.

On the outer edge of the thin part we impose boundary conditions either on the displacements $v_{1}, v_{2}, w$ and angles $\gamma_{1}, \gamma_{2}$ or on the forces $T_{11}, T_{22}, T_{13}, T_{23}$ and momenta $M_{11}, M_{22}$ in the shell (depending if the corresponding parts of the boundary are subjected to load or free). At the outer surface of the shell we prescribe to $\sigma_{i 3}^{+}$and $\sigma_{i 3}^{-}, i=1,2,3$ some given stresses. is:

The operator form of the equations of Timoshenko shell theory

$\mathrm{Ly}=\mathrm{g}$,

with:

$$
\begin{aligned}
& \mathrm{g}=A_{1} A_{2}\left(g_{1}, g_{2}, g_{3}, g_{4}, g_{5}\right)^{T} \\
& g_{1}=p_{1}^{+}+p_{1}^{-} ; g_{2}=p_{2}^{+}+p_{2}^{-} ; g_{3}=p_{3}^{+}-p_{3}^{-} ; \\
& g_{4}=\frac{h}{2}\left(p_{1}^{+}-p_{1}^{-}\right) ; g_{5}=\frac{h}{2}\left(p_{2}^{+}-p_{2}^{-}\right) \\
& \mathrm{y}=\left(\mathrm{v}_{1}, \mathrm{v}_{2}, w, \gamma_{1}, \gamma_{2}\right)^{T}, \mathrm{Ly}=\left(l_{1}, l_{2}, l_{3}, l_{4}, l_{5}\right)^{T} \\
& l_{1}=-\frac{\partial\left(A_{2} T_{11}\right)}{\partial \xi_{1}}+\frac{\partial A_{2}}{\partial \xi_{1}} T_{22}-\frac{1}{A_{1}} \frac{\partial\left(A_{1}^{2} T_{12}\right)}{\partial \xi_{2}}- \\
& \qquad A_{1} A_{2} k_{1} T_{13}-\frac{\partial\left(\frac{A_{1}}{r_{1}} M_{11}\right)}{\partial \xi_{2}}-k_{2} \frac{\partial A_{1}}{\partial \xi_{2}} M_{12}
\end{aligned}
$$


$l_{2}=\frac{\partial A_{1}}{\partial \xi_{2}} T_{11}-\frac{\partial\left(A_{1} T_{22}\right)}{\partial \xi_{2}}-\frac{1}{A_{2}} \frac{\partial\left(A_{2}^{2} T_{12}\right)}{\partial \xi_{1}}-$

$$
-A_{1} A_{2} k_{2} T_{23}-\frac{\partial\left(\frac{A_{2}}{r_{2}} M_{22}\right)}{\partial \xi_{2}}-k_{1} \frac{\partial A_{2}}{\partial \xi_{1}} M_{12}
$$

$l_{3}=A_{1} A_{2} k_{1} T_{11}+A_{1} A_{2} k_{2} T_{22}-\frac{\partial\left(A_{2} T_{13}\right)}{\partial \xi_{1}}-\frac{\partial\left(A_{1} T_{23}\right)}{\partial \xi_{2}}$

$l_{4}=A_{1} A_{2} T_{13}-\frac{\partial\left(A_{2} M_{11}\right)}{\partial \xi_{1}}+\frac{\partial A_{2}}{\partial \xi_{1}} M_{22}-\frac{1}{A_{1}} \frac{\partial\left(A_{1}^{2} M_{12}\right)}{\partial \xi_{2}}$

$l_{5}=A_{1} A_{2} T_{23}+\frac{\partial A_{1}}{\partial \xi_{2}} M_{11}-\frac{\partial\left(A_{1} M_{22}\right)}{\partial \xi_{2}}-\frac{1}{A_{2}} \frac{\partial\left(A_{2}^{2} M_{12}\right)}{\partial \xi_{1}}$

Let us write down the weak formulation of the Timoshenko shell theory problem. Without loss of generality we assume homogeneous boundary conditions. Let us define the following function spaces: $V=\left\{v \in H^{1}\left(\Omega_{2}^{*}\right): v=0, \xi=\left(\xi_{1}, \xi_{2}, \xi_{3}\right) \in\right.$ $\left.\Gamma_{2 D}\right\}, V_{1}=V^{5}$, where $\Gamma_{2 D}$ is the part of the outer boundary of $\Omega_{2}$ on which the kinematic boundary condition is prescribed.

The weak formulation of the problem (4) has the form:

find $\mathrm{y} \in V_{1}$, such that:

$a(\mathrm{y}, \mathrm{v})=g(\mathrm{v}), \forall \mathrm{v} \in V_{1}$,

where: $a(\mathrm{y}, \mathrm{v})=(\mathrm{Ly}, \mathrm{v}), g(\mathrm{v})=(\mathrm{g}, \mathrm{v})$.

Lemma. Assume that for the problem (4) there exist positive constants $r_{10}, r_{20}, A_{10}, A_{20}$ such that:

1. $\left|r_{1}\right| \geq r_{10}>0 ;\left|r_{2}\right| \geq r_{20}>0$;

2. $\left|A_{1}\right| \geq A_{10}>0 ;\left|A_{2}\right| \geq A_{20}>0$

almost everywhere in $\Omega_{2}^{*}$.

Then the bilinear form $a(\mathrm{y}, \mathrm{v})$ for the problem (5) of Timoshenko shell theory is continuous.

Proof. Firstly, we remark that from the assumption 1) it follows that $\left|k_{1}\right| \leq k_{10}<\infty,\left|k_{2}\right| \leq k_{20}<\infty$. Moreover, the assumptions of the lemma do not restrict the class of the problems or the algorithm that can be used for the numerical solution of this problem. Indeed, for the points, for which the assumptions do not hold, the system becomes singular and can no longer be used for the adequate description of the physical process that is being modeled.

The continuity of the bilinear form follows from the fact, that all the coefficients in the system (4) are bounded by modulus almost everywhere, and that the system (4) itself is linear.

The proof also uses the obvious inequality:

$a b \leq \frac{a^{2}+b^{2}}{2}$ for $a, b \in R$.

In order to couple the models in both parts, adequate boundary conditions need to be specified. Let us denote by $\Omega_{2 \text { in }}$ part of the inclusion that lies inside the body $\Omega_{1}$ and by $\Omega_{2 i n}^{*}$ part of the median surface $\Omega_{2}^{*}$ which is the projection of $\Omega_{2 \text { in }}$ on the surface $\xi_{3}=0$.

Let $\Gamma_{I}$ be a boundary, common to both $\Omega_{1}$ and $\Omega_{2}$. Let us divide $\Gamma_{I}$ into the following parts:

$$
\begin{aligned}
& \Gamma_{I_{1}}=\left\{\xi=\left(\xi_{1}, \xi_{2}, \xi_{3}\right):\left(\xi_{1}, \xi_{2}\right) \in \Omega_{2 \text { in }}^{*} ; \xi_{3}=-\frac{h}{2}\right\} \\
& \Gamma_{I_{2}}=\left\{\begin{array}{c}
\xi=\left(\xi_{1}, \xi_{2}, \xi_{3}\right):\left(\xi_{1}, \xi_{2}\right) \in \Omega_{2 i n}^{*} ; \\
\xi_{1}=\xi_{1}^{b} ;-\frac{h}{2} \leq \xi_{3} \leq \frac{h}{2}
\end{array}\right\}
\end{aligned}
$$

$$
\begin{aligned}
& \Gamma_{I_{3}}=\left\{\begin{array}{c}
\xi=\left(\xi_{1}, \xi_{2}, \xi_{3}\right):\left(\xi_{1}, \xi_{2}\right) \in \Omega_{2 i n}^{*} ; \\
\xi_{2}=\xi_{2}^{b} ;-\frac{h}{2} \leq \xi_{3} \leq \frac{h}{2}
\end{array}\right\} \\
& \Gamma_{I_{4}}=\left\{\xi=\left(\xi_{1}, \xi_{2}, \xi_{3}\right):\left(\xi_{1}, \xi_{2}\right) \in \Omega_{2 i n}^{*} ; \xi_{3}=\frac{h}{2}\right\}
\end{aligned}
$$

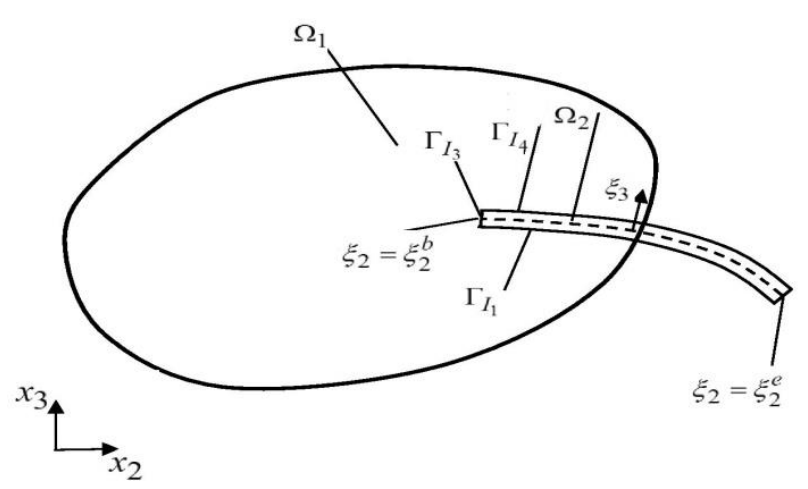

Fig. 2. Cross-section of the body in $\Omega$ by the plane $x_{1}=$ const.

Fig. 2 shows the cross-section of the body in $\Omega$ by the plane $x_{1}=$ const and the connection between rectangular $x_{1}, x_{2}, x_{3}$ and curvilinear $\xi_{1}, \xi_{2}, \xi_{3}$ coordinate systems.

On each part of $\Gamma_{I}$ the following coupling conditions are prescribed (Pelekh, 1978):

- on $\Gamma_{I_{1}}$ (inner part of bottom surface of $\Omega_{2}$ ):

$u_{n}=w, u_{\tau_{1}}=-\mathrm{v}_{1}+\frac{h}{2} \gamma_{1}, u_{\tau_{2}}=-\mathrm{v}_{2}+\frac{h}{2} \gamma_{2}$,

$\sigma_{n n}=-\sigma_{33}^{-}, \sigma_{n \tau_{1}}=-\sigma_{13}^{-}, \sigma_{n \tau_{2}}=-\sigma_{23}^{-}$;

- on $\Gamma_{I_{2}}$ (inner edge of $\Omega_{2}$ ):

$u_{\tau_{1}}=w, u_{\tau_{2}}=-\mathrm{v}_{2}-\xi_{3} \gamma_{2}, u_{n}=\mathrm{v}_{1}+\xi_{3} \gamma_{1}$,

$\int_{-\frac{h}{2}}^{\frac{h}{2}} \sigma_{n n} d \xi_{3}=T_{11}, \quad \int_{-\frac{h}{2}}^{\frac{h}{2}} \sigma_{n \tau_{1}} d \xi_{3}=T_{13}, \quad \int_{-\frac{h}{2}}^{\frac{h}{2}} \sigma_{n \tau_{2}} d \xi_{3}=T_{12}$,

$\int_{-\frac{h}{2}}^{\frac{h}{2}} \sigma_{n n} \xi_{3} d \xi_{3}=M_{11}, \int_{-\frac{h}{2}}^{\frac{h}{2}} \sigma_{n \tau_{2}} \xi_{3} d \xi_{3}=M_{12}$

- on $\Gamma_{I_{3}}$ (inner edge of $\Omega_{2}$ ):

$u_{\tau_{2}}=w, u_{\tau_{1}}=-v_{1}-\xi_{3} \gamma_{1}, u_{n}=v_{2}+\xi_{3} \gamma_{2}$,

$\int_{-\frac{h}{2}}^{\frac{h}{2}} \sigma_{n n} d \xi_{3}=T_{22}, \quad \int_{-\frac{h}{2}}^{\frac{h}{2}} \sigma_{n \tau_{2}} d \xi_{3}=T_{23}, \quad \int_{-\frac{h}{2}}^{\frac{h}{2}} \sigma_{n \tau_{1}} d \xi_{3}=T_{12}$,

$\int_{-\frac{h}{2}}^{\frac{h}{2}} \sigma_{n n} \xi_{3} d \xi_{3}=M_{22}, \int_{-\frac{h}{2}}^{\frac{h}{2}} \sigma_{n \tau_{1}} \xi_{3} d \xi_{3}=M_{12}$

- on $\Gamma_{I_{4}}$ (inner part of top surface surface of $\Omega_{2}$ ):

$u_{n}=-w, u_{\tau_{1}}=\mathrm{v}_{1}+\frac{h}{2} \gamma_{1}, u_{\tau_{2}}=\mathrm{v}_{2}+\frac{h}{2} \gamma_{2}$,

$\sigma_{n n}=-\sigma_{33}^{+}, \sigma_{n \tau_{1}}=\sigma_{13}^{+}, \sigma_{n \tau_{2}}=\sigma_{23}^{+}$.

\section{DOMAIN DECOMPOSITION ALGORITHM}

For the numerical solution of the model domain decomposition algorithm can be used.

The approximate solutions in both domains are connected using Dirichlet-Neumann scheme (Quarteroni and Valli, 1999). Do- 
main decomposition algorithm has the following form:

1. set an initial guess $\lambda^{0}$ for the unknown displacements on the interface $\Gamma_{I}$, set $\varepsilon>0$;

2. for $k=0,1, \ldots$ solve the boundary value problem in $\Omega_{1}$ with the displacements equal to $\lambda^{\mathrm{k}}$ to obtain the approximation for the forces and momenta in $\Omega_{2}$ using (6)-(9);

3. solve the corresponding problem in $\Omega_{2}$ to find the displacements $u_{n}^{k}, u_{\tau_{1}}^{k}, u_{\tau_{2}}^{k}$ on $\Gamma_{I}$;

4. update the displacements $\lambda^{\mathrm{k}}$ on $\Gamma_{I}$ :

- on $\Gamma_{I_{1}}$ :

$\lambda_{11}^{k+1}=(1-\theta) \lambda_{11}^{k}+\theta u_{n}^{k}, \quad \lambda_{12}^{k+1}=(1-\theta) \lambda_{12}^{k}+\theta u_{\tau_{1}}^{k}$, $\lambda_{13}^{k+1}=(1-\theta) \lambda_{13}^{k}+\theta u_{\tau_{2}}^{k}$

- on $\Gamma_{I_{2}}$ :

$\lambda_{21}^{k+1}=(1-\theta) \lambda_{21}^{k}+\theta \mathrm{v}_{1}^{k}$

$\lambda_{23}^{k+1}=(1-\theta) \lambda_{23}^{k}+\theta w^{k}$,

$\lambda_{25}^{k+1}=(1-\theta) \lambda_{25}^{k}+\theta \gamma_{2}^{k}$;

$\lambda_{22}^{k+1}=(1-\theta) \lambda_{22}^{k}+\theta \mathrm{v}_{2}^{k}$, $\lambda_{24}^{k+1}=(1-\theta) \lambda_{24}^{k}+\theta \gamma_{1}^{k}$,

- on $\Gamma_{I_{3}}$ :

$\lambda_{31}^{k+1}=(1-\theta) \lambda_{31}^{k}+\theta \mathrm{v}_{2}^{k}, \quad \lambda_{32}^{k+1}=(1-\theta) \lambda_{32}^{k}+\theta \mathrm{v}_{1}^{k}$,

$\lambda_{33}^{k+1}=(1-\theta) \lambda_{33}^{k}+\theta w^{k}, \quad \lambda_{34}^{k+1}=(1-\theta) \lambda_{34}^{k}+\theta \gamma_{2}^{k}$,

$\lambda_{35}^{k+1}=(1-\theta) \lambda_{35}^{k}+\theta \gamma_{1}^{k}$;

- on $\Gamma_{I_{4}}$ :

$\lambda_{41}^{k+1}=(1-\theta) \lambda_{41}^{k}+\theta u_{n}^{k}$

$\lambda_{42}^{k+1}=(1-\theta) \lambda_{42}^{k}+\theta u_{\tau_{1}}^{k}$,

$\lambda_{43}^{k+1}=(1-\theta) \lambda_{43}^{k}+\theta u_{\tau_{2}}^{k}$,

where $\theta>0$ is a relaxation parameter;

5. if $\left\|\lambda^{\mathrm{k}+1}-\lambda^{\mathrm{k}}\right\| \geq \varepsilon$, then go to step 2, otherwise the algorithm ends.

In the following we assume that the variational problems corresponding to the domains $\Omega_{1}, \Omega_{2}$ and $\Omega$ have unique solutions (Hsiao and Wendland, 2008; Vynnytska and Savula, 2012; Dyyak and Savula, 1997).

Let us prove the convergence of the domain decomposition algorithm and the existence and uniqueness of the solution of the corresponding Steklov-Poincare equation.

For this purpose let us introduce on the common boundary of both domains the function $\varphi \in \Lambda$, with:

$\Lambda=\left\{\varphi=\left(\varphi_{1}, \varphi_{2}, \varphi_{3}, \varphi_{4}\right)\right\}$,

$\varphi_{1}=\left(\varphi_{11}, \varphi_{12}, \varphi_{13}\right), \varphi_{1 i} \in H^{1}\left(\Gamma_{I_{1}}\right), i=1,2,3$;

$\varphi_{2}=\left(\varphi_{21}, \varphi_{22}, \varphi_{23}, \varphi_{24}, \varphi_{25}\right)$,

$\varphi_{2 i}=\varphi_{2 i}\left(\xi_{2}\right) \in H^{\frac{1}{2}}\left(\Gamma_{I_{2}}\right), \quad i=1,2,3,4,5$;

$\varphi_{3}=\left(\varphi_{31}, \varphi_{32}, \varphi_{33}, \varphi_{34}, \varphi_{35}\right)$,

$\varphi_{3 i}=\varphi_{3 i}\left(\xi_{1}\right) \in H^{\frac{1}{2}}\left(\Gamma_{I_{3}}\right), \quad i=1,2,3,4,5$;

$\varphi_{4}=\left(\varphi_{41}, \varphi_{42}, \varphi_{43}\right), \varphi_{4 i} \in H^{1}\left(\Gamma_{I_{4}}\right), i=1,2,3$.

We remark, that the choice of the space $\Lambda$ is motivated by the specifics of the model and is based on the regularity of the corresponding functions on each part of the interface $\Gamma_{I}$.

The connection between the functions $\varphi_{i j}$ and the displacements on the interface is the following:

$$
\begin{aligned}
- & \text { on } \Gamma_{I_{1}}: \\
\varphi_{11} & =u_{n}, \varphi_{12}=u_{\tau_{1}}, \varphi_{13}=u_{\tau_{2}} \\
& - \text { on } \Gamma_{I_{2}}: \\
\varphi_{21} & =\mathrm{v}_{1}, \varphi_{22}=\mathrm{v}_{2}, \varphi_{23}=w, \varphi_{24}=\gamma_{1}, \varphi_{25}=\gamma_{2} ; \\
- & \text { on } \Gamma_{I_{3}}: \\
\varphi_{31} & =\mathrm{v}_{2}, \varphi_{32}=\mathrm{v}_{1}, \varphi_{33}=w, \varphi_{34}=\gamma_{2}, \varphi_{35}=\gamma_{1} ; \\
- & \text { on } \Gamma_{I_{4}}: \\
\varphi_{41} & =u_{n}, \varphi_{42}=u_{\tau_{1}}, \varphi_{43}=u_{\tau_{2}} .
\end{aligned}
$$

Let $\mathrm{S}$ be a Steklov-Poincare operator for our problem and $\mathrm{S}_{i}, i=1,2$ be local Steklov-Poincare operators corresponding to the domains $\Omega_{i}$. Steklov-Poincare operator for the boundaryvalue problem is an operator that transforms boundary conditions of one type into boundary conditions of another type. In our case, Steklov-Poincare operator transforms the displacements on the boundary into loads on the boundary.

Let us multiply interface conditions (6) by $A_{1} A_{2}(1-$ $\left.-k_{1} \frac{h}{2}\right)\left(1-k_{2} \frac{h}{2}\right) ;(7)$ and $(8)$ - by $\frac{1}{h} ;(9)$ - by $A_{1} A_{2}(1+$ $\left.+k_{1} \frac{h}{2}\right)\left(1+k_{2} \frac{h}{2}\right)$.

The Steklov-Poincare operator can be written in the form:

$\{\mathrm{S} \varphi, \psi\}_{\Gamma_{I}}=\left\{\mathrm{S}_{1} \varphi, \psi\right\}_{\Gamma_{I}}+\left\{\mathrm{S}_{2} \varphi, \psi\right\}_{\Gamma_{I}}$, where:

$\left\{\mathrm{S}_{1} \varphi, \psi\right\}_{\Gamma_{I}}=$

$=\left\langle-A_{1} A_{2}\left(1-k_{1} \frac{h}{2}\right)\left(1-k_{2} \frac{h}{2}\right) \sigma_{n n}(\varphi), \psi_{11}\right\rangle_{\Gamma_{I_{1}}}+$

$+\left\langle-A_{1} A_{2}\left(1-k_{1} \frac{h}{2}\right)\left(1-k_{2} \frac{h}{2}\right) \sigma_{n \tau_{1}}(\varphi), \psi_{12}\right\rangle_{\Gamma_{I_{1}}}+$

$+\left\langle-A_{1} A_{2}\left(1-k_{1} \frac{h}{2}\right)\left(1-k_{2} \frac{h}{2}\right) \sigma_{n \tau_{2}}(\varphi), \psi_{13}\right\rangle_{\Gamma_{I_{1}}}+$

$+\left\langle-A_{1} A_{2}\left(1+k_{1} \frac{h}{2}\right)\left(1+k_{2} \frac{h}{2}\right) \sigma_{n n}(\varphi), \psi_{41}\right\rangle_{\Gamma_{I_{4}}}+$

$+\left\langle-A_{1} A_{2}\left(1+k_{1} \frac{h}{2}\right)\left(1+k_{2} \frac{h}{2}\right) \sigma_{n \tau_{1}}(\varphi), \psi_{42}\right\rangle_{\Gamma_{I_{4}}}+$

$+\left\langle-A_{1} A_{2}\left(1+k_{1} \frac{h}{2}\right)\left(1+k_{2} \frac{h}{2}\right) \sigma_{n \tau_{2}}(\varphi), \psi_{43}\right\rangle_{\Gamma_{I_{4}}}+$

$+\left\langle-\frac{1}{h} \int_{-\frac{h}{2}}^{\frac{h}{2}} \sigma_{n n}(\varphi) d \xi_{3}, \psi_{21}\right\rangle_{\Gamma_{I_{2}}}+$

$+\left\langle-\frac{1}{h} \int_{-\frac{h}{2}}^{\frac{h}{2}} \sigma_{n \tau_{2}}(\varphi) d \xi_{3}, \psi_{22}\right\rangle_{\Gamma_{I_{2}}}+$

$+\left\langle-\frac{1}{h} \int_{-\frac{h}{2}}^{\frac{h}{2}} \sigma_{n \tau_{1}}(\varphi) d \xi_{3}, \psi_{23}\right\rangle_{\Gamma_{I_{2}}}+$

$+\left\langle-\frac{1}{h} \int_{-\frac{h}{2}}^{\frac{h}{2}} \sigma_{n n}(\varphi) \xi_{3} d \xi_{3}, \psi_{24}\right\rangle_{\Gamma_{I_{2}}}+$

$+\left\langle-\frac{1}{h} \int_{-\frac{h}{2}}^{\frac{h}{2}} \sigma_{n \tau_{2}}(\varphi) \xi_{3} d \xi_{3}, \psi_{25}\right\rangle_{\Gamma_{I_{2}}}+$

$+\left\langle-\frac{1}{h} \int_{-\frac{h}{2}}^{\frac{h}{2}} \sigma_{n n}(\varphi) d \xi_{3}, \psi_{31}\right\rangle_{\Gamma_{I_{3}}}+$

$+\left\langle-\frac{1}{h} \int_{-\frac{h}{2}}^{\frac{h}{2}} \sigma_{n \tau_{1}}(\varphi) d \xi_{3}, \psi_{32}\right\rangle_{\Gamma_{I_{3}}}+$

$+\left\langle-\frac{1}{h} \int_{-\frac{h}{2}}^{\frac{h}{2}} \sigma_{n \tau_{2}}(\varphi) d \xi_{3}, \psi_{33}\right\rangle_{\Gamma_{I_{3}}}+$

$+\left\langle-\frac{1}{h} \int_{-\frac{h}{2}}^{\frac{h}{2}} \sigma_{n n}(\varphi) \xi_{3} d \xi_{3}, \psi_{34}\right\rangle_{\Gamma_{I_{3}}}+$

$+\left\langle-\frac{1}{h} \int_{-\frac{h}{2}}^{\frac{h}{2}} \sigma_{n \tau_{1}}(\varphi) \xi_{3} d \xi_{3}, \psi_{35}\right\rangle_{\Gamma_{I_{3}}}$ 
$\left\{\mathrm{S}_{2} \varphi, \psi\right\}_{\Gamma_{I}}=$

$=\left\langle-A_{1} A_{2}\left(1-k_{1} \frac{h}{2}\right)\left(1-k_{2} \frac{h}{2}\right) \sigma_{33}^{-}, \psi_{11}\right\rangle_{\Gamma_{I_{1}}}+$

$+\left\langle-A_{1} A_{2}\left(1-k_{1} \frac{h}{2}\right)\left(1-k_{2} \frac{h}{2}\right) \sigma_{13}^{-}, \psi_{12}\right\rangle_{\Gamma_{I_{1}}}+$

$+\left\langle-A_{1} A_{2}\left(1-k_{1} \frac{h}{2}\right)\left(1-k_{2} \frac{h}{2}\right) \sigma_{23}^{-}, \psi_{13}\right\rangle_{\Gamma_{I_{1}}}+$

$+\left\langle-A_{1} A_{2}\left(1+k_{1} \frac{h}{2}\right)\left(1+k_{2} \frac{h}{2}\right) \sigma_{33}^{+}, \psi_{41}\right\rangle_{\Gamma_{I_{4}}}+$

$+\left\langle A_{1} A_{2}\left(1+k_{1} \frac{h}{2}\right)\left(1+k_{2} \frac{h}{2}\right) \sigma_{13}^{+}, \psi_{42}\right\rangle_{\Gamma_{I_{4}}}+\left\langle A_{1} A_{2}(1+\right.$

$\left.\left.+k_{1} \frac{h}{2}\right)\left(1+k_{2} \frac{h}{2}\right) \sigma_{23}^{+}, \psi_{43}\right\rangle_{\Gamma_{I_{4}}}+\left\langle\frac{1}{h} T_{11}, \psi_{21}\right\rangle_{\Gamma_{I_{2}}}+$

$+\left\langle\frac{1}{h} T_{12}, \psi_{22}\right\rangle_{\Gamma_{I_{2}}}+\left\langle\frac{1}{h} T_{13}, \psi_{23}\right\rangle_{\Gamma_{I_{2}}}+\left\langle\frac{1}{h} M_{11}, \psi_{24}\right\rangle_{\Gamma_{I_{2}}}+$

$+\left\langle\frac{1}{h} M_{12}, \psi_{25}\right\rangle_{\Gamma_{I_{2}}}+\left\langle\frac{1}{h} T_{22}, \psi_{31}\right\rangle_{\Gamma_{I_{3}}}+\left\langle\frac{1}{h} T_{12}, \psi_{32}\right\rangle_{\Gamma_{I_{3}}}+$

$+\left\langle\frac{1}{h} T_{23}, \psi_{33}\right\rangle_{\Gamma_{I_{3}}}+\left\langle\frac{1}{h} M_{22}, \psi_{34}\right\rangle_{\Gamma_{I_{3}}}+\left\langle\frac{1}{h} M_{12}, \psi_{35}\right\rangle_{\Gamma_{I_{3}}}$

with $\langle u, v\rangle_{\Gamma_{I}}$ being a bilinear form:

$\langle u, v\rangle_{\Gamma_{I}}=\int_{\Gamma_{I}} u v d \Gamma_{I}, \forall v \in H^{1 / 2}\left(\Gamma_{I}\right), \forall u \in\left(H^{\frac{1}{2}}\left(\Gamma_{I}\right)\right)^{*}$.

Let $\mathrm{Q}, \mathrm{Q}_{1}, \mathrm{Q}_{2}$ be preconditioners of the domain decomposition algorithm for the Dirichlet-Neumann scheme (Quarteroni and Valli, 1999), where: $\mathrm{Q}=\mathrm{Q}_{1}+\mathrm{Q}_{2},\left\{\mathrm{Q}_{1} \varphi, \psi\right\}_{\Gamma_{I}}=\left\{\mathrm{S}_{1} \varphi, \psi\right\}_{\Gamma_{I}}$,

$\left\{Q_{2} \varphi, \psi\right\}_{\Gamma_{I}}=\left\{S_{2} \varphi, \psi\right\}_{\Gamma_{I}}$.

In the case of Dirichlet-Neumann scheme the preconditioners

$\mathrm{Q}, \mathrm{Q}_{1}$ and $\mathrm{Q}_{2}$ coincide with Stelkov-Poincare operators $\mathrm{S}, \mathrm{S}_{1}$ and $\mathrm{S}_{2}$ respectively.

Let us investigate the properties of the Steklov-Poincare operators $S, S_{1}, S_{2}$.

The linearity and symmetry of $S_{2}$ follows directly from the linearity of the corresponding operator in $\Omega_{2}^{*}$, median surface of $\Omega_{2}$. Theorem. Operator $S_{2}$ is continuous and positive-definite on $\Lambda$. Proof. Let us rewrite operator $S_{2}$ in the form:

$\left\{S_{2} \varphi, \psi\right\}_{\Gamma_{I}}=$

$=\int_{\Omega_{2}^{*}} A_{1} A_{2}\left(\left(1+k_{1} \frac{h}{2}\right)\left(1+k_{2} \frac{h}{2}\right) \sigma_{33}^{+}-\left(1-k_{1} \frac{h}{2}\right)(1-\right.$ $\left.\left.-k_{2} \frac{h}{2}\right) \sigma_{33}^{-}\right) \widetilde{w} d \Omega_{2}^{*}+\int_{\Omega_{2}^{*}} A_{1} A_{2}\left(\left(1+k_{1} \frac{h}{2}\right)(1+\right.$

$\left.\left.+k_{2} \frac{h}{2}\right) \sigma_{13}^{+}+\left(1-k_{1} \frac{h}{2}\right)\left(1-k_{2} \frac{h}{2}\right) \sigma_{13}^{-}\right) \tilde{\mathrm{v}}_{1} d \Omega_{2}^{*}+$

$+\int_{\Omega_{2}^{*}} A_{1} A_{2} \frac{h}{2}\left(\left(1+k_{1} \frac{h}{2}\right)\left(1+k_{2} \frac{h}{2}\right) \sigma_{13}^{+}-\left(1-k_{1} \frac{h}{2}\right)(1-\right.$

$\left.\left.-k_{2} \frac{h}{2}\right) \sigma_{13}^{-}\right) \tilde{\gamma}_{1} d \Omega_{2}^{*}+\int_{\Omega_{2}^{*}} A_{1} A_{2}\left(\left(1+k_{1} \frac{h}{2}\right)(1+\right.$

$\left.\left.+k_{2} \frac{h}{2}\right) \sigma_{23}^{+}+\left(1-k_{1} \frac{h}{2}\right)\left(1-k_{2} \frac{h}{2}\right) \sigma_{23}^{-}\right) \tilde{v}_{2} d \Omega_{2}^{*}+$

$+\int_{\Omega_{2}^{*}} A_{1} A_{2} \frac{h}{2}\left(\left(1+k_{1} \frac{h}{2}\right)\left(1+k_{2} \frac{h}{2}\right) \sigma_{23}^{+}-\left(1-k_{1} \frac{h}{2}\right)(1-\right.$

$\left.\left.-k_{2} \frac{h}{2}\right) \sigma_{23}^{-}\right) \tilde{\gamma}_{2} d \Omega_{2}^{*}+\left\langle\frac{1}{h} T_{11}, \tilde{\mathrm{v}}_{1}\right\rangle_{\Gamma_{I_{2}}}+\left\langle\frac{1}{h} T_{12}, \tilde{\mathrm{v}}_{2}\right\rangle_{\Gamma_{I_{2}}}+$

$+\left\langle\frac{1}{h} T_{13}, \widetilde{w}\right\rangle_{\Gamma_{I_{2}}}+\left\langle\frac{1}{h} M_{11}, \tilde{\gamma}_{1}\right\rangle_{\Gamma_{I_{2}}}+\left\langle\frac{1}{h} M_{12}, \tilde{\gamma}_{2}\right\rangle_{\Gamma_{I_{2}}}+$

$+\left\langle\frac{1}{h} T_{22}, \tilde{\mathrm{v}}_{2}\right\rangle_{\Gamma_{I_{3}}}+\left\langle\frac{1}{h} T_{12}, \tilde{\mathrm{v}}_{1}\right\rangle_{\Gamma_{I_{3}}}+\left\langle\frac{1}{h} T_{23}, \widetilde{w}\right\rangle_{\Gamma_{I_{3}}}+$

$+\left\langle\frac{1}{h} M_{22}, \tilde{\gamma}_{2}\right\rangle_{\Gamma_{I_{3}}}+\left\langle\frac{1}{h} M_{12}, \tilde{\gamma}_{1}\right\rangle_{\Gamma_{I_{3}}}$.

Let us substitute the corresponding left-hand sides from the Timoshenko shell theory model equations (4). As a result, we can prove the continuity and coercitivity of the local Steklov-Poincare operator $S_{2}$ taking into account properties of the operator (4). It is known, that the operator (4) is coercive (Vynnytska and Savula, 2008).
Therefore, we obtain:

$\left\{\mathrm{S}_{2} \varphi, \varphi\right\}_{\Gamma_{I}} \geq c^{2} \int_{\Omega_{2}^{*}}\left(\left(\frac{\partial \mathrm{v}_{1}}{\partial \xi_{1}}\right)^{2}+\left(\frac{\partial \mathrm{v}_{2}}{\partial \xi_{1}}\right)^{2}+\left(\frac{\partial w}{\partial \xi_{1}}\right)^{2}+\left(\frac{\partial \gamma_{1}}{\partial \xi_{1}}\right)^{2}+\right.$ $+\left(\frac{\partial \gamma_{2}}{\partial \xi_{1}}\right)^{2}+\left(\frac{\partial \mathrm{v}_{1}}{\partial \xi_{2}}\right)^{2}+\left(\frac{\partial \mathrm{v}_{2}}{\partial \xi_{2}}\right)^{2}+\left(\frac{\partial w}{\partial \xi_{2}}\right)^{2}+\left(\frac{\partial \gamma_{1}}{\partial \xi_{2}}\right)^{2}+\left(\frac{\partial \gamma_{2}}{\partial \xi_{2}}\right)^{2}+$ $\left.+\mathrm{v}_{1}^{2}+\mathrm{v}_{2}^{2}+w^{2}+\gamma_{1}^{2}+\gamma_{2}^{2}\right) d \Omega_{2}^{*}, c \neq 0$.

From (10) it follows that:

$\left\{\mathrm{S}_{2} \varphi, \varphi\right\}_{\Gamma_{I}} \geq \mathrm{c}_{1}^{2} \int_{\Omega_{2}^{*}}\left(\left(-\frac{\partial \mathrm{v}_{1}}{\partial \xi_{1}}+\frac{h}{2} \frac{\partial \gamma_{1}}{\partial \xi_{1}}\right)^{2}+\left(-\frac{\partial \mathrm{v}_{2}}{\partial \xi_{1}}+\right.\right.$ $\left.+\frac{h}{2} \frac{\partial \gamma_{2}}{\partial \xi_{1}}\right)^{2}+\left(-\frac{\partial \mathrm{v}_{1}}{\partial \xi_{2}}+\frac{h}{2} \frac{\partial \gamma_{1}}{\partial \xi_{2}}\right)^{2}+\left(-\frac{\partial \mathrm{v}_{2}}{\partial \xi_{2}}+\frac{h}{2} \frac{\partial \gamma_{2}}{\partial \xi_{2}}\right)^{2}+$ $+\left(\frac{\partial w}{\partial \xi_{1}}\right)^{2}+\left(\frac{\partial w}{\partial \xi_{2}}\right)^{2}+\left(-\mathrm{v}_{1}+\frac{h}{2} \gamma_{1}\right)^{2}+\left(-\mathrm{v}_{2}+\frac{h}{2} \gamma_{2}\right)^{2}+$ $\left.+w^{2}\right) d \Omega_{2}^{*}+c_{2}^{2}\left\|\varphi_{2}\right\|_{H^{\frac{1}{2}\left(\Gamma_{I_{2}}\right)}}^{2}+c_{3}^{2}\left\|\varphi_{3}\right\|_{H^{\frac{1}{2}}\left(\Gamma_{I_{3}}\right)}^{2}+$ $+\mathrm{c}_{4}^{2} \int_{\Omega_{2}^{*}}\left(\left(\frac{\partial \mathrm{v}_{1}}{\partial \xi_{1}}+\frac{h}{2} \frac{\partial \gamma_{1}}{\partial \xi_{1}}\right)^{2}+\left(\frac{\partial \mathrm{v}_{2}}{\partial \xi_{1}}+\frac{h}{2} \frac{\partial \gamma_{2}}{\partial \xi_{1}}\right)^{2}+\left(\frac{\partial \mathrm{v}_{1}}{\partial \xi_{2}}+\right.\right.$ $\left.+\frac{h}{2} \frac{\partial \gamma_{1}}{\partial \xi_{2}}\right)^{2}+\left(\frac{\partial v_{2}}{\partial \xi_{2}}+\frac{h}{2} \frac{\partial \gamma_{2}}{\partial \xi_{2}}\right)^{2}+\left(\frac{\partial w}{\partial \xi_{1}}\right)^{2}+\left(\frac{\partial w}{\partial \xi_{2}}\right)^{2}+$ $\left.+\left(\mathrm{v}_{1}+\frac{h}{2} \gamma_{1}\right)^{2}+\left(\mathrm{v}_{2}+\frac{h}{2} \gamma_{2}\right)^{2}+w^{2}\right) d \Omega_{2}^{*}$,

$c_{i}>0, i=1,2,3,4$.

Therefore, the operator $S_{2}$ is coercive on $\Lambda$.

Let us prove the continuity of $S_{2}$. The continuity of $S_{2}$ follows from the continuity of the operator for the problem (4) in $\Omega_{2}^{*}$.

Using the continuity of the operator for the problem (4), we get:

$\left\{\mathrm{S}_{2} \varphi, \psi\right\}_{\Gamma_{I}} \leq C^{2}\left(\int_{\Omega_{2}^{*}}\left(\left(\frac{\partial \mathrm{v}_{1}}{\partial \xi_{1}}\right)^{2}+\left(\frac{\partial \mathrm{v}_{2}}{\partial \xi_{1}}\right)^{2}+\left(\frac{\partial w}{\partial \xi_{1}}\right)^{2}+\right.\right.$ $+\left(\frac{\partial \gamma_{1}}{\partial \xi_{1}}\right)^{2}+\left(\frac{\partial \gamma_{2}}{\partial \xi_{1}}\right)^{2}+\left(\frac{\partial \mathrm{v}_{1}}{\partial \xi_{2}}\right)^{2}+\left(\frac{\partial \mathrm{v}_{2}}{\partial \xi_{2}}\right)^{2}+\left(\frac{\partial w}{\partial \xi_{2}}\right)^{2}+\left(\frac{\partial \gamma_{1}}{\partial \xi_{2}}\right)^{2}+$ $\left.\left.+\left(\frac{\partial \gamma_{2}}{\partial \xi_{2}}\right)^{2}+v_{1}^{2}+v_{2}^{2}+w^{2}+\gamma_{1}^{2}+\gamma_{2}^{2}\right) d \Omega_{2}^{*}\right)^{1 / 2} \times$

$\times\left(\int_{\Omega_{2}^{*}}\left(\left(\frac{\partial \widetilde{\mathrm{v}}_{1}}{\partial \xi_{1}}\right)^{2}+\left(\frac{\partial \widetilde{\mathrm{v}}_{2}}{\partial \xi_{1}}\right)^{2}+\left(\frac{\partial \widetilde{w}}{\partial \xi_{1}}\right)^{2}+\left(\frac{\partial \widetilde{\gamma}_{1}}{\partial \xi_{1}}\right)^{2}+\left(\frac{\partial \widetilde{\gamma}_{2}}{\partial \xi_{1}}\right)^{2}+\right.\right.$ $+\left(\frac{\partial \widetilde{v}_{1}}{\partial \xi_{2}}\right)^{2}+\left(\frac{\partial \widetilde{v}_{2}}{\partial \xi_{2}}\right)^{2}+\left(\frac{\partial \widetilde{w}}{\partial \xi_{2}}\right)^{2}+\left(\frac{\partial \widetilde{\gamma}_{1}}{\partial \xi_{2}}\right)^{2}+\left(\frac{\partial \widetilde{\gamma}_{2}}{\partial \xi_{2}}\right)^{2}+\widetilde{v}_{1}^{2}+$ $\left.\left.+\tilde{\mathrm{v}}_{2}^{2}+\widetilde{w}^{2}+\tilde{\gamma}_{1}^{2}+\tilde{\gamma}_{2}^{2}\right) d \Omega_{2}^{*}\right)^{1 / 2}, C>0$.

As a result, $\mathrm{S}_{2}$ is continuous.

Let us consider now the local Steklov-Poincare operator $S_{1}$ and rewrite it in the form:

$\left\{\mathrm{S}_{1} \varphi, \psi\right\}_{\Gamma_{I}}=$

$=\left\langle-A_{1} A_{2}\left(1-k_{1} \frac{h}{2}\right)\left(1-k_{2} \frac{h}{2}\right) \sigma_{n n}(\varphi), u_{n}\right\rangle_{\Gamma_{I_{1}}}+$

$+\left\langle-A_{1} A_{2}\left(1-k_{1} \frac{h}{2}\right)\left(1-k_{2} \frac{h}{2}\right) \sigma_{n \tau_{1}}(\varphi), u_{\tau_{1}}\right\rangle_{\Gamma_{I_{1}}}+$

$+\left\langle-A_{1} A_{2}\left(1-k_{1} \frac{h}{2}\right)\left(1-k_{2} \frac{h}{2}\right) \sigma_{n \tau_{2}}(\varphi), u_{\tau_{2}}\right\rangle_{\Gamma_{I_{1}}}+$

$+\left\langle-A_{1} A_{2}\left(1+k_{1} \frac{h}{2}\right)\left(1+k_{2} \frac{h}{2}\right) \sigma_{n n}(\varphi), u_{n}\right\rangle_{\Gamma_{I_{4}}}+$

$+\left\langle-A_{1} A_{2}\left(1+k_{1} \frac{h}{2}\right)\left(1+k_{2} \frac{h}{2}\right) \sigma_{n \tau_{1}}(\varphi), u_{\tau_{1}}\right\rangle_{\Gamma_{I_{4}}}+$

$+\left\langle-A_{1} A_{2}\left(1+k_{1} \frac{h}{2}\right)\left(1+k_{2} \frac{h}{2}\right) \sigma_{n \tau_{2}}(\varphi), u_{\tau_{2}}\right\rangle_{\Gamma_{I_{4}}}+$

$+\left\langle-\frac{1}{h} \int_{-\frac{h}{2}}^{\frac{h}{2}} \sigma_{n n}(\varphi) d \xi_{3}, u_{n}\right\rangle_{\Gamma_{I_{2}}}+$

$+\left\langle\frac{1}{h} \int_{-\frac{h}{2}}^{\frac{h}{2}} \sigma_{n \tau_{2}}(\varphi) d \xi_{3}, u_{\tau_{2}}\right\rangle_{\Gamma_{I_{2}}}+$ 
$+\left\langle-\frac{1}{h} \int_{-\frac{h}{2}}^{\frac{h}{2}} \sigma_{n \tau_{1}}(\varphi) d \xi_{3}, u_{\tau_{1}}\right\rangle_{\Gamma_{I_{2}}}+$

$+\left\langle-\frac{1}{h} \int_{-\frac{h}{2}}^{\frac{h}{2}} \sigma_{n n}(\varphi) d \xi_{3}, u_{n}\right\rangle_{\Gamma_{I_{3}}}+$

$+\left\langle-\frac{1}{h} \int_{-\frac{h}{2}}^{\frac{h}{2}} \sigma_{n \tau_{2}}(\varphi) d \xi_{3}, u_{\tau_{2}}\right\rangle_{\Gamma_{I_{3}}}+$

$+\left\langle\frac{1}{h} \int_{-\frac{h}{2}}^{\frac{h}{2}} \sigma_{n \tau_{1}}(\varphi) d \xi_{3}, u_{\tau_{1}}\right\rangle_{\Gamma_{I_{3}}}$.

Since the Steklov-Poincare operator for the problem of linear elasticity theory is linear, continuous and coercive on $\left(H^{\frac{1}{2}}\left(\Gamma_{I}\right)\right)^{3}$ (Hsiao and Wendland, 2008), and using the continuous and compact embedding $H^{1}(D) \subset \subset H^{\frac{1}{2}}(D)$ for a strong Lipschitz domain $D$ (Hsiao and Wendland, 2008), we get that the operator $\mathrm{S}_{1}$ is linear, continuous and positive on $\Lambda$ (assuming that the corresponding tensions are prescribed as boundary conditions on each part of the interface $\Gamma_{I}$ ).

It is obvious that the Steklov-Poincare operator $\mathrm{S}$ is therefore linear, continuous and coercive.

As a result, the preconditioner operators $Q, Q_{1}$ and $Q_{2}$ are also linear and continuous, and the operators $Q$ and $Q_{2}$ are coercive. Moreover, operator $Q_{2}$ is symmetric.

By Lax-Milgram lemma, the corresponding Steklov-Poincare equation has unique solution.

Let us state the theorem about the convergence of domain decomposition algorithm (Niemi et al., 2010).

Theorem: (the convergence of domain decomposition algorithm).

Let:

- operator $\mathrm{Q}_{2}$ be continuous and coercive on a Hilbert space $X$;

- operator $\mathrm{Q}_{1}$ be continuous on $X$;

- operator $\mathrm{Q}_{2}$ be symmetric and operator $\mathrm{Q}$ be coercive on $X$. Then for arbitrary $\lambda^{0} \in X$ iterations:

$\lambda^{\mathrm{k}+1}=\lambda^{\mathrm{k}}+\theta \mathrm{Q}_{2}^{-1}\left(\mathrm{G}-\mathrm{Q} \lambda^{\mathrm{k}}\right)$

converge in $X$ to the solution of the equation:

$$
\mathrm{Q} \lambda=\mathrm{G}
$$

for arbitrary $\theta$ satisfying $0<\theta<\theta_{\max }$.

Therefore, we have formulated and proven the following

Theorem: Let:

- the Steklov-Poincare operator corresponding to the problem of linear elasticity (1) with corresponding boundary conditions be continuous, symmetric and coercive on the corresponding trace spaces defined in Hsiao and Wendland (2008);

- the assumptions of Lemma hold;

- $A_{1}, A_{2}, k_{1}, k_{2} \in L^{2}\left(\Omega_{2}^{*}\right)$.

- Then the iterative Dirichlet-Neumann scheme for the problem (1)-(4), (6)-(9) with the Dirichlet boundary conditions imposed on the outer edge of the thin part is convergent for some relaxation parameter $\theta$ where $0<\theta<\theta_{\text {max }}$.
Proof: Follows from the theorem about the convergence of domain decomposition algorithms (Dirichlet-Neumann scheme) (Quarteroni and Valli, 1999).

\section{CONCLUSIONS}

We propose a domain decomposition algorithm for the computation of the stress-strain state of the body with thin inclusion. Based on the fact, that the corresponding problems in both parts can be solved separately, one can efficiently solve them preserving the structure and properties of the resulting matrices in both parts. Since the inclusion is modeled using Timoshenko shell theory, the dimension of the problem in the thin part is decreased.

We prove that the corresponding Steklov-Poncare interface equation is well-posed and that the proposed algorithm converges for the appropriately chosen relaxation parameter, which gives the theoretical background for implementation of the proposed algorithm.

\section{REFERENCES}

1. Dyyak I., Savula Ya., Styahar A. (2012), Numerical investigation of a plain strain state for a body with thin cover using domain decomposition, Journal of Numerical and Applied Mathematics, 3 (109), 23-33.

2. Dyyak I., Savula Ya. (1997), D-Adaptive mathematical model of solid body with thin coating, Mathematical Studies, 7 (1), 103-109.

3. Hsiao G.C., Wendland W.L. (2008), Boundary integral equations, Springer.

4. Nazarov S. (2005), Asymptotic analysis and modeling of a jointing of a massive body with thin rods, Journal of Mathematical Sciences, 127 (5), 2192-2262.

5. Niemi A.H., Babuska I., Pitkaranta J., Demkowicz L. (2010), Finite element analysis of the Girkmann problem using the modern hpversion and the classical h-version, ICES Report, 10-47.

6. Pelekh B. (1978), Generalized shell theory, Lviv (in Russian).

7. Quarteroni A., Valli. A. (1999), Domain decomposition methods for partial differential equations, Oxford.

8. Savula Ya., Mang H., Dyyak I., Pauk N. (2000), Coupled boundary and finite element analysis of a special class of two-dimensional problems of the theory of elasticity, Computers and Structures, 75 (2), 157-165.

9. Sulym H. (2007), Bases of mathematical theory of thermoelastic eqiulibrium of deformable solids with thin inclusions, Research and Publishing Center of Shevchenko Scientific Society, Lviv (in Ukrainian).

10. Vynnytska L., Savula Ya. (2008), The stress-strain state of elastic body with thin inclusion, Ph.-Math. Modeling and Information Techonogies, 7, 21-29 (in Ukrainian).

11. Vynnytska L., Savula Ya. (2012), Mathematical modeling and numerical analysis of elastic body with thin inclusion, Computational Mechanics, 50 (5), 533-542. 